Measurement of thermal conductivity and thermal diffusivity using a thermoelectric module

This content has been downloaded from IOPscience. Please scroll down to see the full text.

Download details:

IP Address: 130.133.8.114

This content was downloaded on 26/01/2017 at 11:15

Manuscript version: Accepted Manuscript

Beltrán-Pitarch et al

To cite this article before publication: Beltrán-Pitarch et al, 2017, Meas. Sci. Technol., at press: http://dx.doi.org/10.1088/1361-6501/aa5c30

This Accepted Manuscript is copyright Copyright 2017 IOP Publishing Ltd

During the embargo period (the 12 month period from the publication of the Version of Record of this article), the Accepted Manuscript is fully protected by copyright and cannot be reused or reposted elsewhere.

As the Version of Record of this article is going to be / has been published on a subscription basis, this Accepted Manuscript is available for reuse under a CC BY-NC-ND 3.0 licence after a 12 month embargo period.

After the embargo period, everyone is permitted to use all or part of the original content in this article for non-commercial purposes, provided that they adhere to all the terms of the licence https://creativecommons.org/licences/by-nc-nd/3.0

Although reasonable endeavours have been taken to obtain all necessary permissions from third parties to include their copyrighted content within this article, their full citation and copyright line may not be present in this Accepted Manuscript version. Before using any content from this article, please refer to the Version of Record on IOPscience once published for full citation and copyright details, as permissions will likely be required. All third party content is fully copyright protected, unless specifically stated otherwise in the figure caption in the Version of Record.

When available, you can view the Version of Record for this article at: http://iopscience.iop.org/article/10.1088/1361-6501/aa5c30 


\title{
Measurement of thermal conductivity and thermal diffusivity using a thermoelectric module
}

\author{
Braulio Beltrán-Pitarch ${ }^{1}$, Lourdes Márquez-García ${ }^{2, a}$, Gao Min², Jorge García- \\ Cañadas ${ }^{1, b)}$
}

${ }^{1}$ Department of Industrial Systems Engineering and Design, Universitat Jaume I, Campus del Riu Sec, 12071 Castellón, Spain

${ }^{2}$ Cardiff School of Engineering, Cardiff University, The Parade, Cardiff, CF24 3AA, UK

\begin{abstract}
A proof of concept of using a thermoelectric module to measure both thermal conductivity and thermal diffusivity of bulk disc samples at room temperature is demonstrated. The method involves the calculation of the integral area from an impedance spectrum, which empirically correlates with the thermal properties of the/sample through an exponential relationship. This relationship was obtained employing different reference materials. The impedance spectroscopy measurements are performed in a very simple setup, comprising a thermoelectric module, which is soldered at its bottom side to a $\mathrm{Cu}$ block (heat sink) and thermally connected with the sample at its top side employing thermal grease. Random and systematic errors of the method were calculated for the thermal conductivity (18.6\% and $10.9 \%$, respectively) and thermal diffusivity (14.2\% for both errors) employing a BCR724 standard reference material. Although errors are somewhat high, the technique could be useful for screening purposes or high-throughput measurements at its current state. This new method establishes a new application for thermoelectric modules as thermal properties sensors. It involves the use of a very simple setup in conjunction with a frequency response analyzer, which provides a low cost alternative to most of currently available apparatus in the market. In addition, impedance analyzers are reliable and widely spread equipment, which facilities the sometimes difficult access to thermal conductivity facilities.
\end{abstract}

Keywords: Peltier device, ac impedance, screening, high throughput, thermal properties

a Current address: Department of Industrial Systems Engineering and Design, Universitat Jaume I, Campus del Riu Sec,12071 Castellón, Spain

${ }^{\mathrm{b}}$ Electronic mail: garciaj@uji.es 


\section{INTRODUCTION}

Thermal properties of materials are key in a wide range of applications, such as polymer injection molding, materials for home insulation, heat shielding for space applications, thermal management in electronics, and thermoelectric materials. Within the different thermal properties typically characterized, thermal conductivity $(\lambda)$ is probably the most demanded. Some of the most common methods to measure this property in bulk materials are the laser flash method [1], the guarded hotplate [2], the hot wire [3], and the hot disk [4]. Most of these facilities are very expensive and not readily available in many materials research laboratories, hindering the development of new materials.

In a previous paper [5], we found that the electrical impedance response of a thermoelectric element significantly change when a copper/ceramic plate is attached to it. The variation produced depends on the thermal properties of the copper/ceramic plate. This observation led us to think of the possibility to also observe a significant change in the impedance response of the thermoelement contacted by the copper/ceramic plates (thermoelectric module configuration) if a sample is attached on top of the ceramic. If a new variation induced by the sample is observed, it could be related to the thermal properties of the sample, which could be determined. In addition to this, the possibility to measure the thermal conductivity with a thermoelectric module employing impedance measurements was also pointed out by De Marchi et al. in a previous work [6].

In this paper we have developed the aforementioned idea and we present the proof of concept of a new method to measure room temperature thermal conductivity and thermal diffusivity of disc samples. The method involves the use of a thermoelectric module with the sample mounted on its top and soldered to a heat sink at the bottom. The thermal properties of the sample are determined from the measured impedance spectrum based on an empirical relationship that correlates the integral area of the impedance spectrum to the sample thermal properties. The relationship was established using several reference materials of different thermal properties. The use of a thermoelectric module to measure thermal properties opens up a new application for thermoelectric devices as a thermal property sensor. The simple setup, together with impedance spectroscopy, provides a low cost facility that is more widely available than current commercial apparatus.

\section{EXPERIMENTAL SETUP}

The setup developed in this study (Figure 1) uses a $10 \mathrm{~mm}$ x $10 \mathrm{~mm}$ metallized thermoelectric module (Ref. 048019A30-18RB) from Custom Thermoelectric (USA). The module has 96 legs. The dimension of each leg is $0.6 \mathrm{~mm} \times 0.6 \mathrm{~mm} \times$ $1.05 \mathrm{~mm}$ with $0.5 \mathrm{~mm}$ thick metallized ceramic plates. The area of the module is conveniently chosen to be similar to the area of the sample to be tested. Disc-shaped reference samples with a diameter of $12.7 \mathrm{~mm}$ and thickness of $2.0 \mathrm{~mm}$, covering a 
wide range of thermal conductivity and thermal diffusivity values, were used. Table 1 shows the specifications and thermal properties of the samples employed. Among the samples, a BCR724 standard reference material (SRM) is included and will be used for error evaluation.

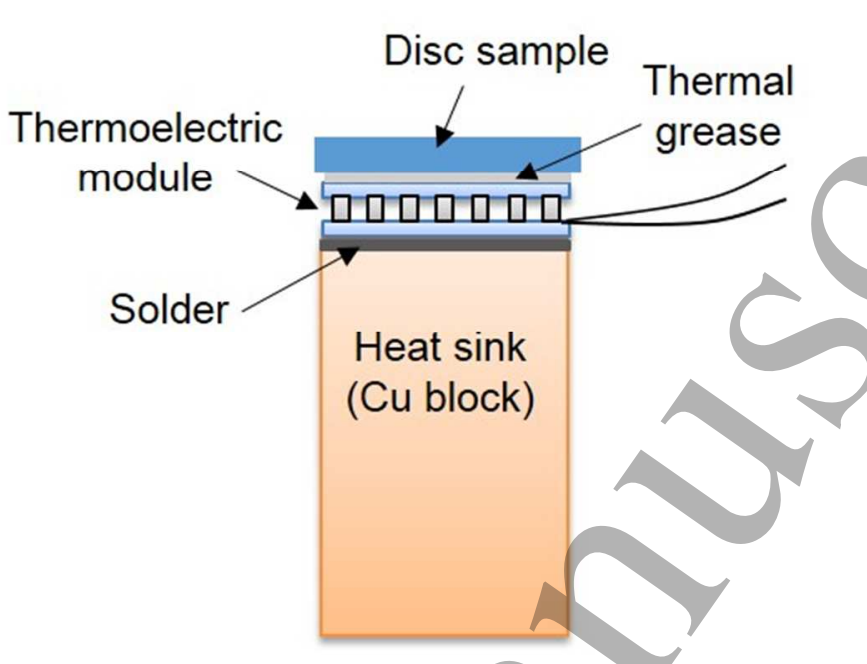

Figure 1. Schematic front view of the experimental setup employed for room temperature thermal conductivity measurements (not to scale).

The sample to be measured was placed on the top side of the thermoelectric module using a thin layer of thermal grease of $2.9 \mathrm{~W} / \mathrm{Km}$ thermal conductivity (Ref. 217-3835) from RS to improve the thermal contact (see Figure 1). For the same purpose, the surface of each sample involved in the thermal contact was polished to a mirror-like finish. This was achieved by first polishing the surfaces with 400,800 and 1200 grit size silicon carbide sandpapers from Buehler. Then, a further polishing was performed with a diamond polishing compound (Metadi II) from Buehler, which was added on a polishing cloth (Texmet 1000, Buehler). After polishing, the surfaces were cleaned with soap and water and finally with acetone just before forming the thermal contact (except PTFE which reacts with acetone).

Table 1. Specifications and thermal properties at room temperature of the disc samples evaluated. Values were provided from the different suppliers, except when marked with $\left(^{*}\right)$ which were obtained from alternative sources. All samples diameter and thickness is 12.7 mm and $2 \mathrm{~mm}$ respectively.

\begin{tabular}{|c|c|c|c|c|c|}
\hline Sample & Sunnl & $\begin{array}{c}\text { Alloy } \\
\text { identification }\end{array}$ & $\begin{array}{l}\text { Density } \\
\left(\mathrm{g} / \mathrm{cm}^{3}\right)\end{array}$ & $\begin{array}{c}\text { Thermal conductivity } \\
(\mathrm{W} / \mathrm{Km})\end{array}$ & $\begin{array}{c}\text { Thermal diffusivity } \\
\left(\mathrm{mm}^{2} / \mathrm{s}\right)\end{array}$ \\
\hline PTFE & Broncesval (Spain) & Virgin & 2.18 & 0.25 & $0.11 *$ \\
\hline Pyrex & Netzsch (UK) & $\begin{array}{l}\text { Reference } \\
\text { material }\end{array}$ & 2.22 & 1.10 & 0.65 \\
\hline BCR-724 & $\begin{array}{c}\text { LGC Standards } \\
\text { (UK) }\end{array}$ & SRM & 2.48 & 4.06 & 1.89 \\
\hline Stainless Steel & Asucer (Spain) & AISI 304 & $7.8^{*}$ & $14.00 *$ & $3.77 *$ \\
\hline Bronze & Broncesval (Spain) & B-10 & 8.7 & 50.63 & $15.31 *$ \\
\hline Aluminium & Broncesval (Spain) & $\mathrm{Cu}$ alloy 2030 & 2.8 & 135.00 & $54.94 *$ \\
\hline
\end{tabular}


The bottom side of the module was soldered to a $\mathrm{Cu}$ block $(10 \mathrm{~mm} \times 10 \mathrm{~mm}$ x $50 \mathrm{~mm})$ acting as a heat sink, which significantly improves the repeatability of the method. The soldering was performed using a conventional solder (Pb60Sn40 of $\sim 50 \mathrm{~W} / \mathrm{Km}$ thermal conductivity) with a hot plate as heat source and following the indications from the supplier [7]. To facilitate the temperature propagation from the hot plate during the soldering process, the $\mathrm{Cu}$ block was surrounded by aluminium foil. The final thickness of the solder layer was $\sim 240 \mu \mathrm{m}$.

Impedance measurements were performed at room conditions inside a Faraday cage using an Autolab PGSTAT30 potentiostat equipped with a FRA2 impedance analyzer module. The frequency range ( $2 \mathrm{kHz}$ to $2 \mathrm{mHz}$ ), which comprises the part of the signal lying in the $Z^{\prime} /-Z^{\prime \prime}$ quadrant, was kept fixed for all measurements. A total of 40 points logarithmically distributed were always recorded. A $20 \mathrm{~mA}$ ac current amplitude was employed and experiments were performed at 0 A dc value. Each measurement takes around $30 \mathrm{~min}$ to be completed.

\section{THERMAL PROPERTIES CORRELATION}

The impedance response of all the samples from Table 1 can be seen in Figure 2. The response without sample is also shown as reference. For the samples with lower thermal conductivity/diffusivity values (Figure 2a), it can be seen clearly that the integral area below the impedance spectra increases with increasing thermal conductivity/diffusivity (from PTFE to stainless steel). The increase occurs mainly on the low frequency part (i.e., right part of the spectra). These results reveal a possible correlation between the integral area and the thermal conductivity/diffusivity of the sample, and an empirical relationship was obtained as shown in Figure 3.

For the samples with higher thermal conductivity/diffusivity values (Figure 2b), the variation in the integral area is less significant, especially for thermal conductivities/diffusivities above the value of bronze. For these samples, an increase in the area with increasing thermal conductivity/diffusivity occurs in both the high (inset of Figure $2 b$ ) and low frequency parts (Figure $2 b$ ), in contrast to the case of Figure 2a, where the area decreases at the high frequency part (inset of Figure 2a). Due to the small differences in the integral area between bronze and aluminium samples, it is difficult to obtain a sensitive correlation with the thermal properties in this range, as shown in Figure 3.

Based on the knowledge obtained from our previous work [8], we can perform a qualitative analysis in order to understand the different impedance responses observed in Figure 2. In general, three different regions can be distinguished in most of the spectra. The first region appears at the highest frequencies ( $2 \mathrm{kHz}$ to $\sim 20 \mathrm{~Hz}$, with $\mathrm{Z}^{\prime}$ values from 2.9 to $\sim 2.95 \Omega$ ) and it is very similar for all the samples, as observed in the insets of Figure 2. This high frequency part is governed by the 
1

2

3

4

5

6

7

8

9

10

11

12

13

14

15

16

17

18

19

20

21

22

23

24

25

26

27

28

29

30

31

32

33

34

35

36

37

38

39

40

41

42

43

44

45

46

47

48

49

50

51

52

53

54

55

56

heat diffusion along the ceramic layers after heat generation/absorption at the junctions (Peltier effect), without noticing the presence of the sample or $\mathrm{Cu}$ block. This is due to the higher thermal conductivity of the ceramics $(\sim 35 \mathrm{~W} / \mathrm{Km})$ respect to the thermoelements $(\sim 1.5 \mathrm{~W} / \mathrm{Km})$, which produces a minor diffusion towards the latter [8].
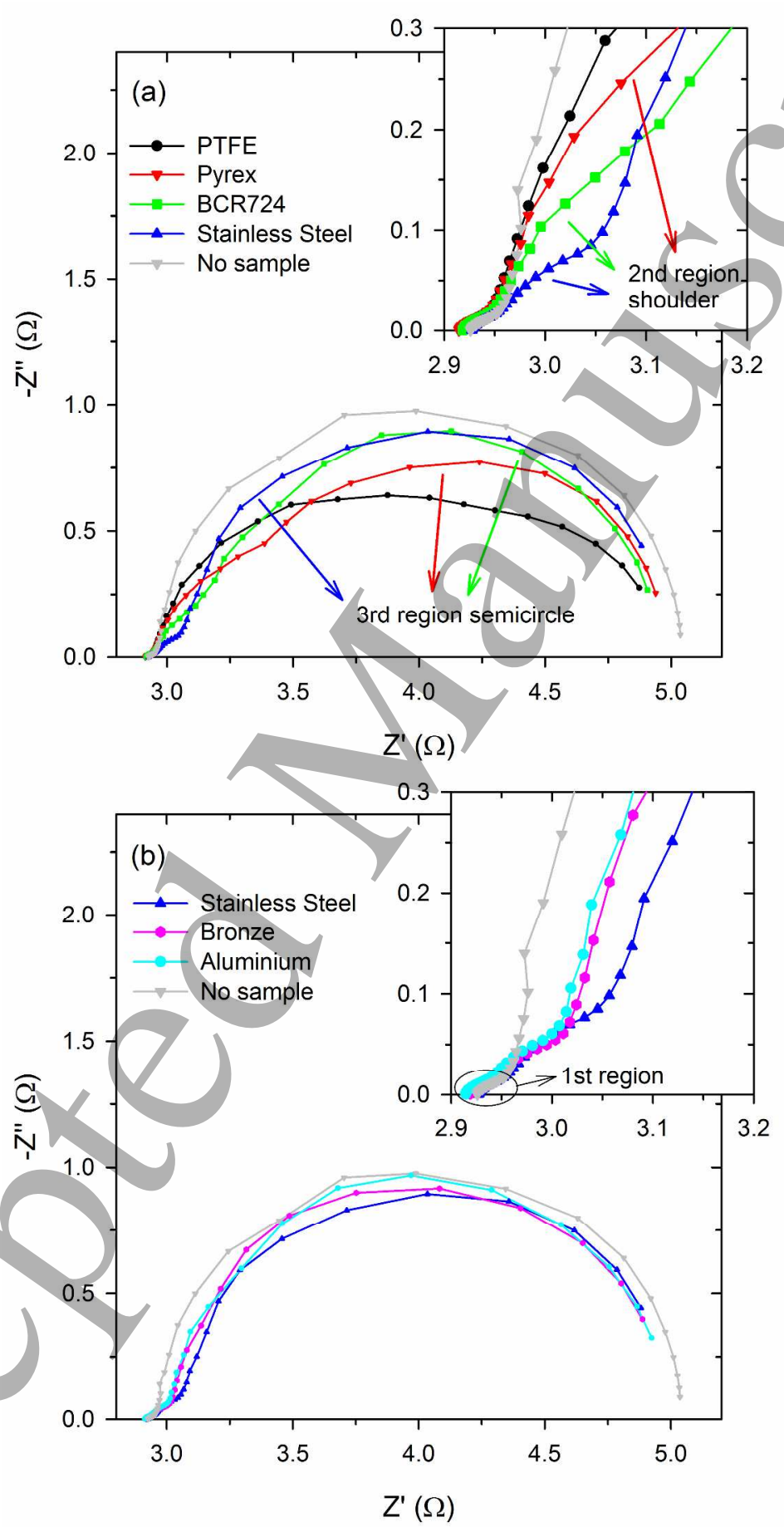

Figure 2. Impedance spectra obtained for the disc samples in Table 1. The samples with lower thermal conductivity values are shown in (a). The samples with higher thermal conductivity values are presented in (b). The insets represent a zoom to the high frequency part. The frequency decreases from left to right. The spectrum obtained without sample is also include as reference. 
The second region appears at reduced frequencies, around the middle section of the spectra, where the events taking place at the ceramic layer attached to the sample are responsible for the changes observed, since the ceramic layer soldered to the $\mathrm{Cu}$ block is a common part to all measurements. In this region, a shoulder (more clearly seen for the stainless steel sample at $Z^{\prime}$ values from 2.95 to $3.05 \Omega$ ) can be observed. This shoulder increases and displaces towards lower frequencies (right side) when moving from high to low thermal conductivities/diffusivities (aluminium to PTFE), as more significantly shown in Figure 2a. At this second region (shoulder), the diffusion of heat, once reached the end of the ceramic, takes place in the sample and also towards the thermoelements. When heat diffusion is quicker in the sample (samples with higher thermal conductivity) the shoulder less significantly overlaps with the final semicircle part (third region), which is the case of the samples in Figure 2b. For the samples with the lowest thermal conductivity (PTFE and Pyrex), the shoulder significantly spreads and overlaps with the final semicircle part. It should be noted that the heat can diffuse along the sample until its end is reached. When this happens heat transport can no longer progress and the heat is mainly absorbed (accumulated) in the sample rather than transported. The change from diffusion to accumulation control takes place around the end of the shoulder, which is clearly reached at higher frequencies (quicker) for higher thermal conductivity samples.

The third region comprises the final semicircle, related to the cooling experienced around the half length of the thermoelements [8], produced by the cold flux coming from the cold junction, which occurs simultaneously to the heat accumulation (absorption) in the sample and hot side ceramic. In cases where the heat accumulation still does not dominate at this low frequency region, due to the slow heat diffusion along the sample, this third part is significantly altered (as the case for the lowest thermal conductivity samples) by the overlap with the shoulder. It should be noticed that this competition between thermal transport and heat accumulation in the sample, occurring at similar frequencies than the cooling effect from the cold junction, is the main event responsible for the integral area variations observed.

As a summary and from a more general point of view, the way the Peltier heat evolves from the junctions, which depends on the thermal properties of all the elements (sample, ceramics and thermoelements), is what influences the impedance response. Diffusion involves both the transport and absorption of heat, which is accounted for in the thermal diffusivity definition $\left(\alpha=\lambda / \rho C_{p}\right)$, determined by the thermal conductivity and the volume specific heat $\left(\rho C_{p}\right.$, being $\rho$ the mass density and $C_{p}$ the specific heat). A dominant heat accumulation in the system, which occurs when the diffusion is completed (end of the sample reached) rises the impedance response (increases the imaginary part). Hence, the quicker the heat transport from the hot junction to the end of the sample (higher sample thermal conductivity/diffusivity), the larger the integral area, 
1

2

3

4

5

6

7

8

9

since the heat accumulation becomes dominant sooner (at higher frequencies). This is what occurs in the spectrum without sample, which exhibit the larger area since the diffusion is completed quicker (end of the ceramic plate).

The integral of each measurement was calculated in order to quantitatively establish the correlation between the thermal properties and the area $A$ below the impedance spectra (Figure 3). Three impedance measurements were performed for each sample with remade thermal contact to evaluate repeatability. The correlation of all the measurements with the thermal conductivity and thermal diffusivity can be observed in Figure 3a and 3b, respectively. As previously mentioned from the measurements in Fig. $2 \mathrm{~b}$, for samples with large thermal conductivity/diffusivity $\left(\lambda>14 \mathrm{~W} / \mathrm{Km}, \alpha>3.8 \mathrm{~mm}^{2} / \mathrm{s}\right)$ a loss in sensitivity is observed, since the differences in the integral area for the two samples with higher thermal conductivity/diffusivity are very small, which produces the change observed in the trend of the curves (saturation) and makes difficult to determine the thermal properties in this range. However, for the rest of the samples $\left(\lambda<14 \mathrm{~W} / \mathrm{Km}, \alpha<3.8 \mathrm{~mm}^{2} / \mathrm{s}\right)$, an exponential relationship is clearly observed and the experimental points were fitted in order to obtain the empirical correlation equations,

$$
\begin{aligned}
& \lambda=\exp \left(-13.062+11.560 A^{2}\right), \\
& \alpha=\exp \left(-12.165+10.038 A^{2}\right) .
\end{aligned}
$$

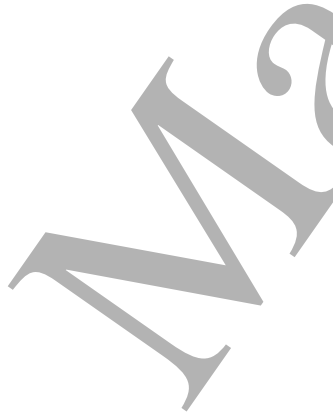

The fitting was performed using statistical weighting for both the thermal property and integral area data and excluding the measurements from the BCR724 SRM, which will be used later for the error evaluation. The above equations allow the determination of the thermal conductivity and diffusivity from the experimentally calculated integral area of the impedance spectra. It should be noticed that the parameters obtained from the fitting can differ under different conditions, such as when samples of different geometries are used, or if a different thermoelectric module is employed. However, they can be obtained by repeating the outlined calibration procedure under the different conditions.

\section{ERROR ANALYSIS}

In order to evaluate the accuracy of the new method for the determination of both thermal properties $(\lambda$ and $\alpha)$, the random and systematic errors were calculated using the BCR724 SRM. Three measurements with remade thermal contacts were performed to obtain a mean value for the integral area and its standard deviation $\left(1.259 \pm 0.013 \Omega^{2}\right)$. The mean value is 
represented in Figures $3 \mathrm{a}$ and $3 \mathrm{~b}$ vs. the corresponding true value of thermal conductivity and thermal diffusivity, respectively, showing a close approximation to the predicted value (solid line).

The total combined random error $u_{c}$, was calculated taking into account different contributions: (i) the standard deviation from the three measurements in the SRM $\left(0.013 \Omega^{2}\right)$, (ii) the standard errors (95\% confidence) obtained from the corresponding fitting (both $\mathrm{i}$ and ii are represented by $u\left(x_{i}\right)$ ), and (iii) the interdependence of the fitted parameters $u\left(x_{i}, x_{j}\right)$, provided by the covariance matrix. The equation employed to determine $u_{c}$ for the thermal conductivity was [9],

$$
u_{c}^{2}=\sum_{i=1}^{N}\left(\frac{\partial \lambda}{\partial x_{i}}\right)^{2} u^{2}\left(x_{i}\right)+2 \sum_{i=1}^{N-1} \sum_{j=i+1}^{N} \frac{\partial \lambda}{\partial x_{i}} \frac{\partial \lambda}{\partial x_{j}} u\left(x_{i}, x_{j}\right)
$$
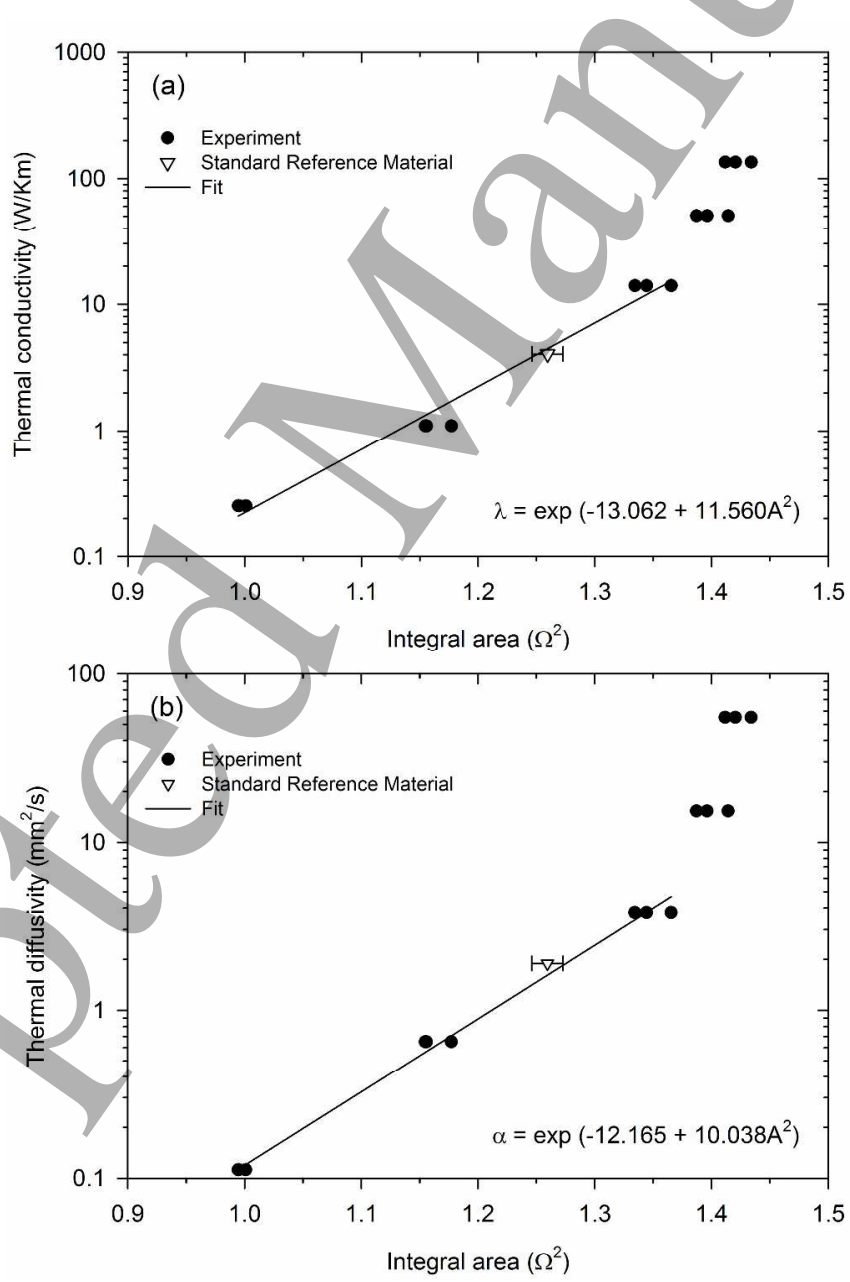

Figure 3. Empirical correlations of the integral area (A) obtained from the impedance spectra of the disc samples from Table 1 with (a) their thermal conductivity and (b) their thermal diffusivity values. Three measurements were performed for each sample (dots). Lines indicate the fit to the experimental points for the samples with lower thermal conductivity/diffusivity. Fit equations are also given. The mean value from 3 measurements of the BCR724 standard reference material respect the certified values (triangles) are also included. The error bar represents the standard deviation. 
The same equation using thermal diffusivity derivatives $\partial \alpha / \partial\left(x_{i}, x_{j}\right)$ replacing $\partial \lambda / \partial\left(x_{i}, x_{j}\right)$ was used for the thermal diffusivity. The $\partial(\lambda, \alpha) / \partial x_{i}, u\left(x_{i}\right), u\left(x_{i}, x_{j}\right)$ and calculated $u_{c}$ values are shown in Table 2 . Total combined random errors above 18 and $14 \%$ were obtained for $\lambda$ and $\alpha$, respectively, which are somewhat high. This is to a great extent due to the exponential relationship between the thermal properties and the integral area, which provides an order of magnitude higher $\partial(\lambda, \alpha) / \partial A$ values with respect to the derivatives of the fitted parameters $(\partial(\lambda, \alpha) / \partial a$ and $\partial(\lambda, \alpha) / \partial b)$, as shown in Table 2 . This increases the total combined error in despite of the good repeatability in the experimental determination of $A(1.1 \%)$. We would like to remark that especially the outer edge of the sample could not be completely under adiabatic conditions and radiation and convection phenomena could be taking place. However, due to the small current amplitude used in the impedance measurements $(20 \mathrm{~mA})$, no large temperature differences with respect to the ambient temperature were found (for the stainless steel sample a maximum temperature difference of $1.8{ }^{\circ} \mathrm{C}$ was obtained, which occurred only at low frequencies), which will not provide a significant influence of radiation/convection phenomena. It should be also noticed that especial care should be taken to keep the thermal grease layer thin, since it was estimated that the integral area can increase up to $\sim 7 \%$ if the thermal grease layer is thick.

Table 2. Mean predicted values obtained from three measurements performed for BCR724 standard reference material, derivatives respect to the variables affected by errors, standard deviation $u(\mathrm{~A})$ for the integral area $A$, the standard errors $u(a, b)$ from the fitting parameters to the general equation $\lambda, \alpha=\exp (a+b A)$, their interdependence $u\left(x_{i}, x_{j}\right)$, and the total combined random error $u_{c}$, calculated for both the thermal conductivity and thermal diffusivity. Percentage values are given between brackets).

\begin{tabular}{|c|c|c|c|c|c|c|}
\hline Thermal property & Mean value & $x_{i}$ & $\partial(\lambda, \alpha) / \partial x_{i}$ & $u\left(x_{i}\right)$ & $u\left(x_{i}, x_{i}\right)$ & $\boldsymbol{u}_{\boldsymbol{c}}$ \\
\hline$x_{0}$ & $4.50 \mathrm{~W} / \mathrm{Km}$ & & $\begin{array}{c}51.63 \mathrm{~W} / \Omega^{2} \mathrm{Km} \\
4.47 \mathrm{~W} / \mathrm{Km} \\
5.63 \mathrm{~W} \Omega^{2} / \mathrm{Km}\end{array}$ & $\begin{array}{c}0.013 \Omega^{2}(1.1 \%) \\
0.704(5.4 \%) \\
0.606 \Omega^{-2}(5.2 \%)\end{array}$ & $-0.424 \Omega^{2}$ & $0.839 \mathrm{~W} / \mathrm{Km}(18.6 \%)$ \\
\hline$\alpha$ & $1.62 \mathrm{~mm}^{2} / \mathrm{s}$ & & $\begin{array}{c}16.17 \mathrm{~mm}^{2} / \Omega^{2} \mathrm{~s} \\
1.61 \mathrm{~mm}^{2} / \mathrm{s} \\
2.03 \mathrm{~mm}^{2} \Omega^{2} / \mathrm{s}\end{array}$ & $\begin{array}{c}0.013 \Omega^{2}(1.1 \%) \\
0.327(2.7 \%) \\
0.281 \Omega^{-2}(2.8 \%)\end{array}$ & $-0.091 \Omega^{2}$ & $0.230 \mathrm{~mm}^{2} / \mathrm{s}(14.2 \%)$ \\
\hline
\end{tabular}

The systematic error was calculated comparing the mean value obtained from the three measurements of the BCR724 SRM using the empirical equations for the thermal conductivity (Equation 1) and thermal diffusivity (Equation 2), with respect to the true values $\left(4.09 \mathrm{~W} / \mathrm{Km}\right.$ and $1.89 \mathrm{~mm}^{2} / \mathrm{s}$ respectively). Systematic errors of $0.44 \mathrm{~W} / \mathrm{Km}(10.9 \%)$ and 0.28 $\mathrm{mm}^{2} / \mathrm{s}(14.7 \%)$ were obtained, which are somewhat lower than the random error for the case of the thermal conductivity and very similar for the thermal diffusivity. Due to the high errors identified, the method could be useful at its current state for screening purposes and high-throughput measurements. The time to complete one impedance measurement is $\sim 30$ min, and the experimental setup does not require a complicated preparation of the sample, which only has to be placed at the top of the thermoelectric module after spreading a layer of thermal grease. 
We would like to remark that despite the high errors, they are not very far from the errors of many of the available facilities in the market. The main purpose of this article is to provide a demonstration of the proof of concept and it is likely to improve accuracy by future developments. On the other hand, the current method only applies to disc samples with 12.7 $\mathrm{mm}$ diameter and $2 \mathrm{~mm}$ thickness. However, by performing new correlations using reference samples with different dimensions, new empirical equations adapted to other sample sizes could be easily found. Moreover, the measurements could be extended to higher temperatures when required by varying the ambient temperature with a band heater or a furnace. The highest temperature possible will be limited by the maximum operating temperature of the module.

\section{CONCLUSIONS}

A proof of concept of a new method to determine both thermal conductivity and thermal diffusivity of disc samples has been demonstrated. The technique consists in the determination of the integral area from an impedance spectrum. It was found that this area follows an empirical exponential relationship with the thermal conductivity/diffusivity of the sample. A calibration equation, obtained using different reference materials, allows the direct determination of the thermal conductivity/diffusivity from the measured integral area. The impedance spectroscopy measurements are performed in a thermoelectric module, which is soldered at the bottom to a Cu block and contacted with the sample at the top employing thermal grease. Random and systematic errors around 19 and $11 \%$ were observed for the thermal conductivity, respectively, employing a standard reference material. For the thermal diffusivity around 14 and $15 \%$ values were found, respectively. Main uncertainty contributions to the random errors are attributed to the exponential nature of the calibration equation. Despite the large errors, the technique could be useful for screening purposes and high throughput measurements. The new method establishes a new application for thermoelectric modules as thermal property sensor. It involves the use of a very simple setup connected to a frequency response analyzer, which presents a low cost alternative to most of commercial equipment available in the market. In addition, reliable impedance analyzers are widely spread, which facilities the sometimes difficult access to thermal conductivity facilities.

\section{ACKNOWLEDGMENTS}

The authors wish to acknowledge financial support from the Ramón y Cajal programme (RYC-2013-13970) and the Accelerated Metallurgy Project, which is co-funded by the European Commission in the 7th Framework Programme (contract NMP4-LA-2011-263206), by the European Space Agency and by the individual partner organizations. The experimental support of Raquel Oliver Valls and José Ortega Herreros is also acknowledged. 


\section{REFERENCES}

[1] Parker W J, Jenkins R J, Butler C P and Abbott G L 1961 Flash Method of Determining Thermal Diffusivity, Heat Capacity, and Thermal Conductivity J. Appl. Phys. 321679

[2] Salmon D 2001 Thermal conductivity of insulations using guarded hot plates, including recent developments and sources of reference materials Meas. Sci. Technol. 12 R89-98

[3] Assael M J, Antoniadis K D and Wakeham W A 2010 Historical Evolution of the Transient Hot-Wire Technique Int. J. Thermophys. 31 1051-72

[4] He Y 2005 Rapid thermal conductivity measurement with a hot disk sensor Thermochim. Acta 436 122-9

[5] García-Cañadas J and Min G 2014 Impedance spectroscopy models for the complete characterization of thermoelectric materials J. Appl. Phys. 116174510

[6] De Marchi A and Giaretto V 2014 The Peltier driven frequency domain approach in thermal analysis Rev. Sci. Instrum. 85103904

[7] http://www.customthermoelectric.com/TECmounting.html, accessed on 3 November 2016.

[8] García-Cañadas J and Min G 2016 Thermal dynamics of thermoelectric phenomena from frequency resolved methods AIP Adv. 635008

[9] 1995 Evaluation of measurement data - GUM: Guide to the expression of uncertainty in measurement 